\title{
Splenic lipidosis after administration of intravenous fat emulsions
}

\author{
G. B. FORBES
}

From the Department of Pathology, Kent and Canterbury Hospital, Canterbury, Kent CT1 3NG, UK

SUMMARY Spleens showing fatty infiltration and necrosis of the pulp were found at necropsy on several patients who had received intravenous fat emulsions during their terminal illnesses. The postmortem findings are described and the clinicopathological correlation is discussed with special reference to the phenomenon of creaming of the emulsion.

Intravenous fat emulsions provide a concentrated source of calories in a relatively low volume of fluid, and their use, combined with other forms of parenteral nutrition, is indicated in a wide variety of medical and surgical conditions. They are particularly valuable as an energy source in malnourished patients with disease of the alimentary tract (Hadfield, 1965) and in patients with acute or chronic renal failure (Lee et al., 1967). Refinements in the preparation of lipid for parenteral use have produced an emulsion that will provide an intake of over $\mathbf{2 0 0 0}$ calories per litre in the form of synthetic chylomicrons, which are basically of similar size, composition, and physiological behaviour to natural chylomicrons. The newer emulsions, and particularly the soyabean-egg yolk phosphatide emulsion (Intralipid), are well tolerated by man, and side effects are said to be infrequent and trivial when the emulsion is administered in the recommended dosage (Wretlind, 1974).

Numerous studies have been carried out on the physiological and pathological effects of repeated fat emulsion administered to experimental animals and to man, and these have been reviewed comprehensively by Thompson (1974). Studies on human beings have been confined largely to the living subject, and there are few reports of postmortem findings on patients who had received intravenous lipid therapy before death. The findings of several authors, for example Lee (1974), were negative or minimal in respect of postmortem abnormalities that could have been attributed to the infusions. However, Bozian et al. (1957) found large amounts of intravascular fat in the lungs, liver, spleen, and kidneys of one patient who had received 7 litres of cotton-seed oil emulsion

Received for publication 30 December 1977 immediately before death, and the findings were regarded as 'similar to those observed in patients dying after fat embolism'; necropsies on four other lipid-treated patients in this series showed slight to moderate fatty infiltration only.

In a series of experiments, Elster (1970) studied morphological changes in the organs of animals and man after intravenous nutrition with various fat emulsions. He noted massive accumulation of fat in the lungs, liver, spleen, and kidneys of dogs given deliberately excessive dosage, and fatty infiltration of the spleen was accompanied by distinctive degeneration of the organ, which he termed 'spodogenic splenic tumour'. He reported similar but less intense changes in the organs of seven necropsied human beings; in one patient the spleen was pale and of doughy consistency, and sections showed pronounced fatty degeneration of the pulp.

Freund et al. (1975) reported their necropsy findings on a patient with iatrogenic lipidosis after a course of intravenous lipid emulsion (Intralipid). The spleen was moderately enlarged and pale pink, and microscopic examination revealed an infiltration of fat in this organ as well as in the liver, kidneys, and lungs.

\section{Present study}

In the course of routine necropsy practice in a general hospital during 1975 and 1976, when approximately 1500 necropsies were performed, a spleen of unusually pale appearance was encountered on 12 occasions. The significance of this finding was not appreciated at first, especially as the patients had died from some obvious unrelated primary cause. When several spleens with this peculiar appearance had been seen it was decided to review the clinical and postmortem material and to investigate the 
nature of the lesion. It soon became apparent that the factor common to all cases was the administration of lipid emulsions (Intralipid) during the patient's terminal illness, and microscopic examination of subsequent specimens, using fat-specific stains, showed fatty infiltration of the spleen, liver, and, to a lesser extent, kidneys and other organs.

\section{MACROSCOPIC FEATURES}

The macroscopic appearance of the spleens is summarised in Table 1. It will be seen that there was

Table 1 Morbid anatomy of spleens

\begin{tabular}{|c|c|c|c|c|}
\hline \multirow[t]{2}{*}{ Case } & \multirow[t]{2}{*}{ Size $(g)$} & \multirow[t]{2}{*}{ Colour } & \multicolumn{2}{|c|}{ Histology } \\
\hline & & & Fat & Necrosis \\
\hline 1 & 220 & Salmon-pink & NT & +++ \\
\hline 2 & 370 & NR & NT & $++t$ \\
\hline 3 & Small & Pale & $++t$ & + \\
\hline 4 & Small & Pale & +++ & $+t+$ \\
\hline 5 & 825 & Salmon-pink & NT & +++ \\
\hline 6 & 205 & Very pale & NT & +++ \\
\hline 7 & Normal & Pale & NT & + \\
\hline 8 & NR & NR & +++ & +++ \\
\hline 9 & 200 & Putty & +++ & $++t$ \\
\hline 10 & 75 & Very pale & ++ & $++t$ \\
\hline 11 & 190 & Pale & ++ & + \\
\hline 12 & 210 & Pale & + & $+t+$ \\
\hline
\end{tabular}

$\mathrm{NR}=$ not recorded $; \mathrm{NT}=$ not specifically tested.

considerable variation in the size of the organ from a minimum of $75 \mathrm{~g}$ to a maximum of $825 \mathrm{~g}$, but most specimens showed little deviation from the normal weight range of 150 to $200 \mathrm{~g}$. All spleens were distinctly pale, and an occasional specimen was salmonpink or putty-coloured (Fig. 1). The consistency of the transected specimen was moderately soft in the majority of cases, but on no occasion was it diffluent or of 'septic' appearance. There was no evidence of thromboembolism of the splenic vessels at the hilum, and infarctions of conventional type were not observed. The necropsies were performed within 45 hours of death, most within 24 hours, and postmortem autolysis was consistently absent or insignificant.

\section{MICROSCOPIC FEATURES}

In sections stained with haematoxylin and eosin the whole of the splenic pulp was seen to be acellular and degenerate, and only the capsule, septa, and a few follicles were preserved in recognisable form (Fig. 2); in most cases the degeneration amounted to a diffuse massive necrosis. The pulp was bloodless, cell outlines and nuclei were blurred or absent, and in a few cases fat was detectable as rounded empty spaces (Fig. 3). The presence of an infiltration of macroglobules, microglobules, and particulate fat was confirmed when frozen sections were stained specifically for fat using the oil-red 0 technique (Fig.흐 4). The degree of necrosis was roughly proportional to that of fatty infiltration, but exceptions to this were noted in two cases: case 3 showed heavy fato infiltration and moderate necrosis, and these findings were reversed in case 12 . Unfortunately, fat was not $\frac{\bar{\rho}}{\frac{1}{\sigma}}$ looked for specifically in five spleens as I was aware $\stackrel{\mathbb{Q}}{\circ}$ too late of the possible association between the giving of fat emulsion and the abnormal spleens, by which time the postmortem specimens had been. discarded and one was left with paraffin-embedded $\overrightarrow{\vec{\omega}}$ material only.

Other organs and tissues were examined micro- $\frac{}{0}$ scopically. The necrotic changes seen in the spleens $\dot{\omega}$ were not in evidence in any other organ, with the $\overrightarrow{+}$ possible exception of the liver of case 11, sections of $-v$ which showed small scattered foci of degeneration. The livers of all patients treated with Intralipid $\mathrm{O}$ showed moderate to marked fatty infiltration, a not unexpected finding as the liver plays a major role in the removal of emulsified fat from the bloodstreame and its subsequent storage. The fat was sometimes $\stackrel{5}{\rightarrow}$ readily visible in haematoxylin and eosin sections as $\vec{\varphi}$ well as in oil-red 0 sections, and it was of similar appearance and distribution to that seen in fatty infiltration from other causes, for example, alcohol abuse. Intravenous fat pigment, as described by Thompson (1974) and Passwell et al. (1976), was not detected in sections of livers and spleens; the $\stackrel{2}{\circ}$ deposition of this pigment is usually the result of $\stackrel{\mathbb{Q}}{\circ}$ long-term lipid administration and is probably of no $\overline{\overrightarrow{0}}$ consequence.

In one patient large globules and emboli of fat were found microscopically within the lumina of blood vessels throughout the body, but this patient was receiving Intralipid at the time of death, and the cadaver blood was grossly hyperlipaemic on nakedeye examination. This fatty appearance of the blood, especially of the clotted blood in the heart chambers, was seen in a few other patients who had received Intralipid shortly before death, and, when pleural and peritoneal effusions were present, these too were $\frac{D}{0}$ of chylous appearance. In passing, one might add that gross hyperlipaemia is frequently seen in blood $N$ samples from live patients on lipid infusions, and this can cause troublesome technical problems in hae- $N$ matological and biochemical tests in the laboratory, $\omega$ for example, erroneously high haemoglobin levels (Nicholls, 1977).

\section{CLINICAL CORRELATION}

A summary of the relevant clinical details of the necropsied patients is given in Table 2. All patients were middle-aged or elderly, and there was no significant sex preponderance. The majority of patients $\triangle$ were suffering from severe renal failure but this 


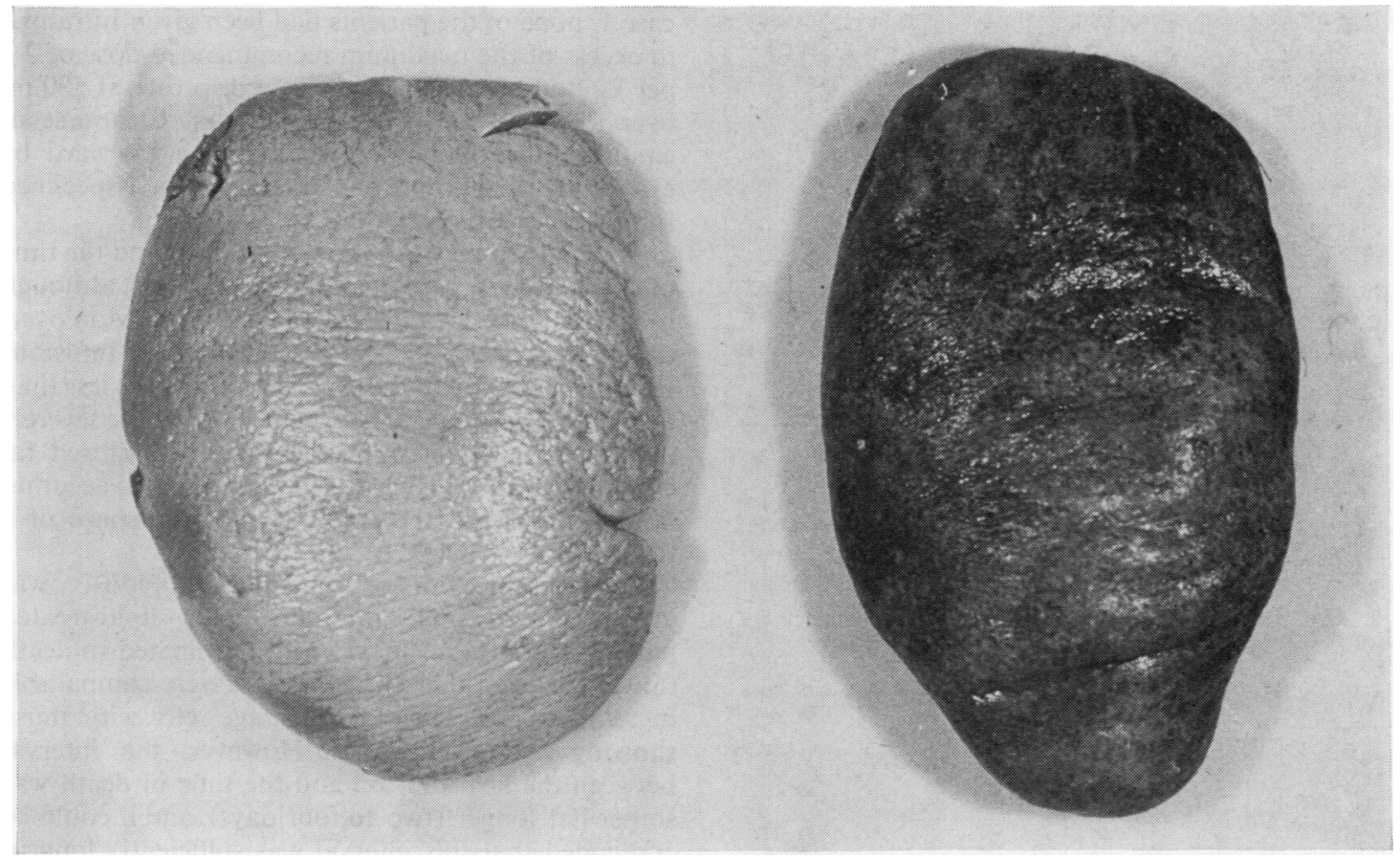

Fig. 1 Case 9. Putty-like spleen (left) with normal spleen (right) for comparison.

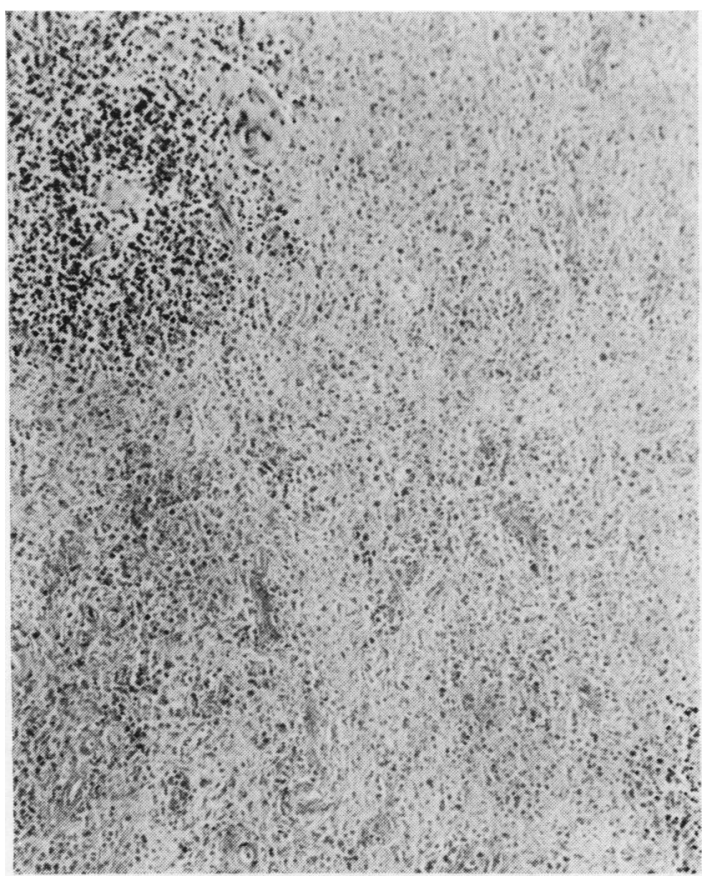

Fig. 2 Case 12. Section of spleen showing necrosis of pulp. (Haematoxylin and eosin $\times 95$ )

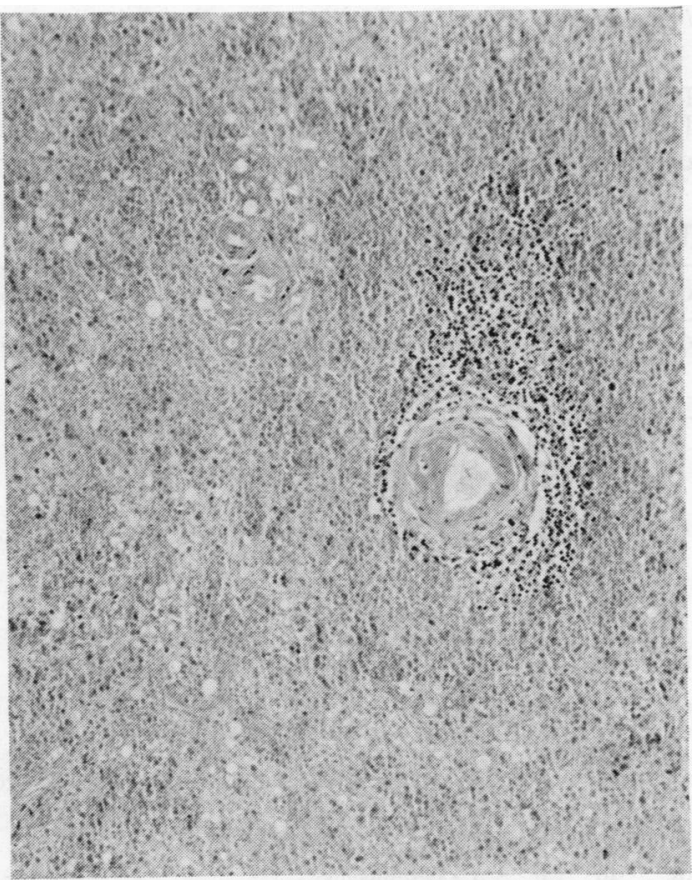

Fig. 3 Case 8. Section of spleen showing necrosis and fat vacuoles. ( $H$ and $E \times 95$ ) 


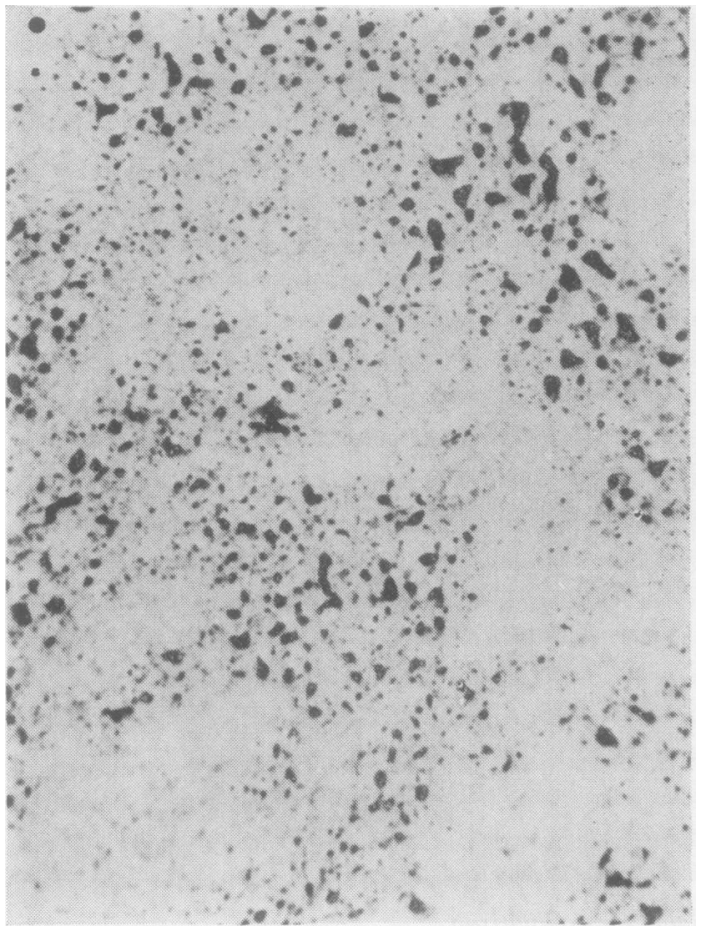

Fig. 4 Case 10. Section of spleen showing fatty infiltration. (Oil-red $0 \times 160$ )

probably reflects the much more frequent use of Intralipid in the renal ward by comparison with the other hospital wards. With the possible exception of case 1 , none of the patients had been given Intralipid in excess of the maximum recommended dose of $2 \mathrm{~g}$ per $\mathbf{k g}$ body weight per day at a drip rate of $500 \mathrm{ml}$ over a period of six to eight hours. Clearance of $\bar{g}$ emulsion from the blood was tested in the ward by examining the plasma of a morning blood specimen for absence of turbidity before reinfusion.

The interval between the last infusion and the time of death in most cases was 12 hours or less, although in one instance the interval was five days. Moreover, the period of terminal illness during which infusions were given was (with the exception of case 5) less than nine days. These observations suggest that the severely ill patient is unable to metabolise the infused fat $\dot{\omega}$ completely, and this results in a progressive accumulation of lipid in the spleen within the space of a few days.

Postmortem evidence of splenic lipidosis was absent or minimal in four other lipid-treated patients with severe illness which terminated in death (lower part of Table 2); these cases were comparable ce in terms of age, diagnosis, dosage, etc, with those $\stackrel{5}{\rightarrow}$ showing marked lipidosis. However, the interval $\vec{\theta}$ between the last infusion and the time of death was somewhat longer (two to four days), and it could be contended that this interval was sufficiently long to allow elimination of most of the fat from the spleen.

\section{Discussion}

The present study is based for the most part on retrospective postmortem findings, and there is a bias in the material towards patients who died from renal

Table 2 Clinicopathological correlation

\begin{tabular}{|c|c|c|c|c|c|c|c|c|c|}
\hline \multicolumn{4}{|c|}{ Clinical data } & \multicolumn{4}{|c|}{ Intralipid infusion } & \multicolumn{2}{|l|}{ Spleen } \\
\hline Case & Age & Sex & Main cause of death & $\begin{array}{l}\text { Strength } \\
(\%)\end{array}$ & $\begin{array}{l}\text { Volume } \\
\text { (litres) }\end{array}$ & $\begin{array}{l}\text { Period of } \\
\text { treatment } \\
\text { (days) }\end{array}$ & $\begin{array}{l}\text { Interval } \\
\text { before death } \\
\text { (hours) }\end{array}$ & Fat & Necrosis \\
\hline 1 & 48 & $\mathbf{M}$ & CGN & 20 & 7 & 4 & $<12$ & NT & $++t$ \\
\hline 2 & 46 & $\mathbf{M}$ & Staph. septicaemia & 20 & $2 \cdot 5$ & 4 & 30 & NT & +++ \\
\hline 3 & 39 & $\mathbf{M}$ & CGN (transplant) & 20 & 3 & 6 & $<12$ & $++t$ & \\
\hline 4 & 68 & $\mathbf{F}$ & CGN & 20 & $4 \cdot 5$ & 9 & $<12$ & $++t$ & $+t+$ \\
\hline 5 & 44 & $\mathbf{F}$ & CGN (diabetic) & $10+20$ & 14 & 28 & $<12$ & NT & $++t$ \\
\hline 6 & 50 & $\mathbf{M}$ & CGN & 10 & 2.5 & 2 & $<12$ & NT & +++ \\
\hline 7 & 65 & $\mathbf{M}$ & CGN & 20 & 0.5 & $\overline{1}$ & $<12$ & NT & \\
\hline 8 & 70 & $\mathbf{M}$ & Uraemia, ca bladder & 20 & $4 \cdot 5$ & 5 & 120 & +++ & +++ \\
\hline 9 & 74 & $\mathbf{F}$ & Ca stomach & 10 & $2 \cdot 5$ & 4 & $<12$ & +++ & $++t$ \\
\hline 10 & 66 & $\mathbf{F}$ & $\begin{array}{l}\text { Acute renal failure, cerebral } \\
\text { embolism }\end{array}$ & 10 & $3 \cdot 5$ & 3 & $<12$ & ++ & ++ \\
\hline $\begin{array}{l}11 \\
12\end{array}$ & $\begin{array}{l}54 \\
69\end{array}$ & $\begin{array}{l}\mathbf{M} \\
\mathbf{M}\end{array}$ & $\begin{array}{l}\text { CGN } \\
\text { Ureteric obstruction, ca rectum }\end{array}$ & $\begin{array}{l}20 \\
10\end{array}$ & $\begin{array}{l}2 \\
5 \cdot 5\end{array}$ & $\begin{array}{r}4 \\
13\end{array}$ & $\begin{array}{l}12 \\
30\end{array}$ & $+t$ & $\stackrel{+}{+}+$ \\
\hline & & & & & & & & & \\
\hline 13 & 65 & $\mathbf{M}$ & CGN (diabetic), cerebral thrombosis & $10+20$ & 3 & 7 & 72 & NT & \pm \\
\hline 14 & 66 & $\mathbf{F}$ & Polycystic kidneys & 20 & $2 \cdot 5$ & 5 & 96 & \pm & - \\
\hline 15 & 47 & $\mathbf{M}$ & $\begin{array}{l}\text { CGN (transplant), Aspergillus } \\
\text { meningitis }\end{array}$ & 10 & 4 & 8 & 48 & $\bar{\mp}$ & - \\
\hline 16 & 70 & $\mathbf{F}$ & Ca oesophagus & 20 & 1 & 1 & 60 & $\mp$ & - \\
\hline
\end{tabular}

CGN = chronic glomerulonephritis; - Negative; $N T=$ not specifically tested. 
failure. The conclusion to be drawn at this stage is that some, but not all, severely ill patients show intolerance to fat emulsions administered during their terminal illness, resulting in a distinctive splenopathy, which could be termed iatrogenic splenic lipidosis. This occasional adverse reaction is disquieting but it is of very doubtful significance as a contributing factor to the death of the patient. Moreover, it must be set against the great benefit derived by thousands of patients whose well-being, and indeed survival, have depended on the high nutritive value of lipid emulsions.

The incidence of splenic lipidosis is difficult to assess but it is probably quite low in relation to the widespread use of lipid emulsions, as judged by the paucity of recorded examples of this complication in the world literature. It is all the more surprising, therefore, that 12 examples of this splenopathy should have been encountered in one hospital in the space of two years. At a rough estimate this represents an incidence of $5 \%$ of approximately 200 patients given a total of 835 litres of Intralipid during the period of study. We are unable to offer any ready explanation for this relatively high local incidence other than the already mentioned fact that the patients given emulsions were severely ill and some were in a moribund condition. The technique of administration and other relevant factors were investigated and were found to be no different from those pertaining in other hospitals and renal units. It is of interest in this connection that the clinicians at this hospital, alerted to the possible risk of fatoverloading on the evidence of postmortem findings, have been using Intralipid during the past six months in smaller quantities and mostly at $10 \%$ strength, and it is perhaps more than coincidence that only one case of lipidosis has been seen at necropsy during this latter period when the emulsion has been used more circumspectly.

The fate of infused lipid emulsion is thought to be similar to that of fat which enters the bloodstream after oral ingestion. Experiments in animals and man have shown that the lipid phase of the emulsion, in the form of triglycerides, is hydrolysed to free fatty acids in the blood and cells through the agency of the enzyme lipoprotein lipase (clearing factor). There is thought to be a rapid but temporary accumulation of fat in the reticuloendothelial cells of the liver and spleen. This lasts for up to 24 hours, and the lipid is then recirculated in the blood stream to be metabolised quickly or stored in various organs and tissues for later utilisation. The storage of infused fat is a function that devolves largely on the liver, skeletal muscle, adipose tissue, and other organs, including the spleen.

The foregoing sequence of events applies to emulsions that are kinetically stable, and the stabilising factor in most modern lipid emulsions is the phosphatide, which forms the outer shell of the chylomicron and which, like the lipid phase, is eventually metabolised. Much work has gone into perfecting a stable lipid emulsion for safe therapeutic use, and the present-day commercial preparations, including Intralipid, are considered to meet the accepted requirements of stability. However, if the emulsion is physically unstable, or if the patient's condition alters the emulsion so that it becomes unstable, flocculation and coalescence of the chylomicrons can occur in the recipient's blood; this interferes with the clearance mechanism as the fat is less easily assimilated in this form (Geyer, 1970).

In 1961 LeVeen et al., using the cotton-seed oil emulsion Lipomul, made several important clinical observations relating to the stability of the emulsion in vitro and in vivo. They found that all human beings did not handle fat emulsions in the same way and that the rate of clearance varied considerably from patient to patient. They attributed this to the ability of some patients to cream or break the emulsion, and they found that the sera of $70 \%$ of randomly selected patients creamed Lipomul in vitro to a greater or less extent, and that debilitated patients tended to cream the emulsion more readily than well patients. They also made the important observation that creamers cleared fat from the bloodstream more rapidly than non-creamers as a result of flocculation and embolisation of the chylomicrons. Later studies by LeVeen et al. (1965) showed that the factor in the blood responsible for creaming was a gamma globulin, serum calcium acting in an ancillary capacity. Geyer (1970) has confirmed that flocculation can occur with all the modern commercially available emulsions but noted that the phenomenon was minimal with $10 \%$ Intralipid.

We have carried out experiments on a small scale in our laboratory in order to satisfy ourselves of the validity and possible significance of these observations. The technique used to detect creaming was essentially the same as that used by LeVeen et al. (1961) except that Intralipid was used as the test emulsion; $0.05 \mathrm{ml}$ of emulsion was added to $2 \mathrm{ml}$ of serum and, after gentle mixing, the tubes were kept at room temperature and examined at three, six, and 18 hours. Creaming was readily detectable to the naked eye in the form of a white supernatant layer overlying clear serum; in the absence of creaming the mixture remained homogenous and opalescent (Fig. 5). Creaming, when heavy, usually became visible within six hours but an interval of 18 hours was usually required for the test to become strongly positive; however, the reaction could be speeded up by incubating the tubes in a water-bath at $37^{\circ} \mathrm{C}$. 


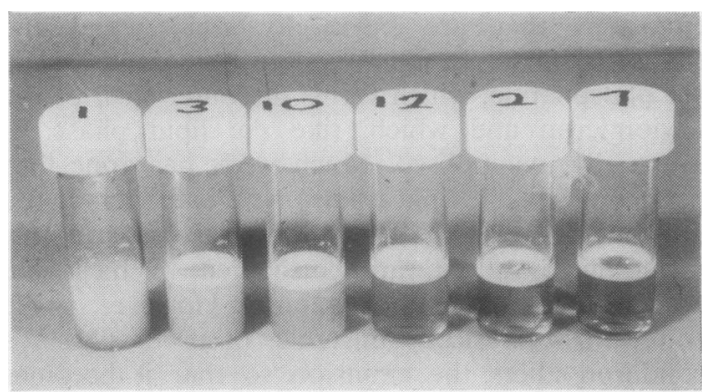

Fig. 5 In vitro creaming of Intralipid by sera from random hospital patients. The tubes have been arranged to show gradations from absence of creaming (tube 1) to complete creaming (tubes 12, 2, and 7).

Sera from 48 subjects, none of whom was receiving Intralipid, were tested for their ability to cream the emulsion. Twelve were ambulant outpatients, and the serum of only one outpatient (suffering from rheumatoid arthritis) creamed the emulsion. By contrast, six of 12 randomly selected inpatients showed creaming to a greater or less extent; the tests on these inpatients were read without foreknowledge of their clinical condition, and when this was ascertained it was found that positive results were obtained with sera from patients who were far from well, the degree of positivity being roughly proportional to the severity of illness. An additional 12 patients with advanced renal disease were tested, and seven of them creamed the emulsion to a greater or less extent. In this group, creaming was apparently unrelated to the blood urea level as one patient with a normal blood urea $(5.0 \mathrm{mmol} / \mathrm{l} ; 30$ $\mathrm{mg} / 100 \mathrm{ml}$ ) creamed the emulsion heavily, and a patient with a blood urea level of $34.0 \mathrm{mmol} / \mathrm{l}$ (204 $\mathrm{mg} / 100 \mathrm{ml}$ ) was a non-creamer. Finally, 12 healthy subjects (laboratory staff) were tested, and the results were negative. Table 3 summarises the results of this preliminary experiment; they confirm the findings of LeVeen $e t$ al. and, contrary to Geyer's experience, we found that creaming occurred readily with Intralipid at both $10 \%$ and $20 \%$ strengths.

The further observation by LeVeen et al. that creamers clear the infused fat from the bloodstream more quickly that non-creamers is of practical importance when ward-testing the patient's postinfusion plasma for fat clearance. It is the current practice to regard a fat-free supernatant plasma on visual inspection as indicating clearance of the emulsion and a safety signal for repeating the infusion should this be required. If the patient is a creamer, however, this test would simply indicate rapid clearance from the circulating blood but not necessarily from the body, and repeated infusions to
Table 3 Tests for creaming

\begin{tabular}{|c|c|c|c|}
\hline Source & No. tested & Creamers & Non-creamer \\
\hline Outpatients & 12 & 1 & 11 \\
\hline Random inpatients & 12 & 6 & 6 \\
\hline Renal patients & 12 & 7 & 5 \\
\hline Healthy controls & 12 & 0 & 12 \\
\hline
\end{tabular}

such a patient could lead to fat-overloading of $\vec{\circ}$ receptive organs. On this assumption one might $\vec{\omega}$ postulate that the abnormal spleens seen at necropsyo were the result of creaming of the emulsion and죵 subsequent overloading of the organ, the build-up of $\omega$ lipid then causing a gradual obstruction of the blood $\vec{\infty}_{\infty}$ circulation within the sinusoids, leading to an inci- pient ischaemic necrosis of the pulp. The unique ${ }^{\circ}$ vasculature of the spleen might account for the을 observation that necrosis was found only in thisorgan. This hypothesis cannot be substantiated on present evidence as the creaming test was carried outo during life on only one of the patients (case 12) whon later came to necropsy. The result of the test on this $\vec{\varphi}$ patient was positive at the 18-hour reading but not strongly so $(+)$. It is also interesting to speculate on the possible after-effects of heavy fat-overloading ofo the spleen should the patient survive. Is the damage of a temporary or permanent nature, and is ito responsible for depressed antibody formation and\% the increased susceptibility of patients on fat $\stackrel{\mathbb{Q}}{2}$ emulsions to develop infection and septicaemia? $\overrightarrow{\overrightarrow{0}}$

The high proportion of patients suffering from 3 renal failure in the present series calls for brief? comment although, as already stated, the frequent use of lipid emulsions in the renal ward offers a probable explanation for this preponderance in the clinical material. The kidneys play little part in the 3 metabolism and excretion of either natural or artificial chylomicrons, and fat-overloading in renalo patients is therefore unlikely to be due directly to impaired kidney function. However, Lawson (1965) has shown that renal failure delays the rate of elimination of infused fat on the evidence of raised?. serum triglyceride levels, and he attributed this to N saturation of available binding sites on the albumin molecule by organic substances which had accumu- $N$ lated as a result of renal disease. Lee et al. (1967) dow not consider this to be a contraindication to the use? of lipid emulsions, and indeed they stress the beneficial effect of these infusions in the management of renal failure as the lipid provides a large number of ${ }^{-}$ calories without the accumulation of nitrogenous $-\frac{T}{T}$ breakdown products. Fat overloading, a side effect $\stackrel{+}{\mathbb{D}}$ of the earlier fat emulsions, was not encountered by $\stackrel{\odot}{\square}$ Lee et al. in their patients given the then newer $\unrhd$ emulsion, Intralipid, but my own necropsy experience 
challenges this assumption, at least in regard to the spleen.

\section{Conclusion}

During a two-year period of hospital necropsy work 12 patients, who had received Intralipid infusion during their terminal illnesses, showed a splenopathy which was characterised by lipid infiltration and necrosis of the pulp. It would seem that severe illness reduces the stability of the emulsion with consequent fat overloading, and this may be related to the propensity of ill patients to cream the fat. Further study is needed to assess the clinical significance of this finding; in the meantime clinicians are advised to exercise caution when prescribing intravenous lipid emulsions to severely ill patients, especially to patients whose sera readily flocculate the emulsion on prior in-vitro testing.

I thank my clinical colleagues, especially those in the renal ward, for their willing and helpful cooperation. I also received much help and advice from the Stockholm and London branches of KabiVitrum Ltd, the manufacturers of Intralipid. I am most grateful to Mr E. W. Spice, FIMLS, for technical and photographic assistance, and to Valerie Halliday for secretarial assistance.

\section{References}

Bozian, R. C., Davidson, N. W., Stutman, L. J., and Wilkinson, C. F., Jr. (1957). Observations on the use of intravenous fat emulsions in man. Metabolism, 6, 703716.

Elster, K. (1970). Morphological changes after intravenous nutrition with fat emulsions. In Parenteral Nutrition, edited by H. C. Meng and D. H. Law, pp. 434-439. Charles C. Thomas, Springfield, Illinois.
Freund, U., Krausz, Y., Levij, I. S., and Eliakim, M. (1975). Iatrogenic lipidosis following prolonged intravenous hyperalimentation. American Journal of Clinical Nutrition, 28, 1156-1160.

Geyer, R. P. (1970). Parenteral emulsions-formulation, preparation and use in animals. In Parenteral Nutrition, edited by H. C. Meng and D. H. Law, pp. 339-375. Charles C. Thomas, Springfield, Illinois.

Hadfield, J. I. H. (1965). Preoperative and postoperative intravenous fat therapy. British Journal of Surgery, 52, 291-298.

Lawson, L. J. (1965). Parenteral nutrition in surgery. British Journal of Surgery, 52, 795-800.

Lee, H. A. (1974). Post mortem examinations. In Parenteral Nutrition in Acute Metabolic Illness, edited by H. A. Lee, pp. 419-420. Academic Press, London and New York.

Lee, H. A., Sharpstone, P., and Ames, A. C. (1967). Parenteral nutrition in renal failure. Postgraduate Medical Journal, 43, 81-91.

LeVeen, H. H., Giordano, P., and Johnson, A. (1965). Flocculation of intravenous fat emulsions. American Journal of Clinical Nutrition, 16, 129-134.

LeVeen, H. H., Giordano, P., and Spletzer, J. (1961). The mechanism of removal of intravenously injected fat. Archives of Surgery, 83, 311-321.

Nicholls, P. D. (1977). The erroneous haemoglobinhyperlipaemia relationship. Journal of Clinical Pathology, 30, 638-640.

Passwell, J. H., David, R., Katznelson, D., and Cohen, B. E. (1976). Pigment deposition in the reticuloendothelial system after fat emulsion infusion. Archives of Disease in Childhood, 51, 366-368.

Thompson, S. W. (1974). The Pathology of Parenteral Nutrition with Lipids. Charles C. Thomas, Springfield, Illinois.

Wretlind, A. (1974). Fat emulsions. In Parenteral Nutrition in Acute Metabolic Illness, edited by H. A. Lee, pp. 77-95. Academic Press, London and New York.

Requests for reprints to: Dr G. B. Forbes, Department of Pathology, Kent and Canterbury Hospital, Canterbury CT1 3NG. 